\title{
HARNACK ESTIMATES FOR CONJUGATE HEAT KERNEL ON EVOLVING MANIFOLDS
}

\author{
XIAODONG CAO, HONGXIN GUO, AND HUNG TRAN
}

\begin{abstract}
In this article we derive Harnack estimates for conjugate heat kernel in an abstract geometric flow. Our calculation involves a correction term $\mathcal{D}$. When $\mathcal{D}$ is nonnegative, we are able to obtain a Harnack inequality. Our abstract formulation provides a unified framework for some known results, in particular including corresponding results of Ni [8, Perelman [10] and Tran [12] as special cases. Moreover it leads to new results in the setting of Ricci-Harmonic flow and mean curvature flow in Lorentzian manifolds with nonnegative sectional curvature.
\end{abstract}

\section{Contents}

1. Introduction

1.1. Main Results

2. Preliminaries

2.1. Evolution Equations

2.2. Asymptotic Behavior and Reduced Geometry 4

2.3. Entropy Formulas

3. Estimates on the Heat Kernel

3.1. A Gradient Estimate

3.2. $L_{\infty}$ Bound

3.3. Proofs of Main Results

References

\section{INTRODUCTION}

Assume that $M$ is an $n$-dimensional closed manifold endowed with a one-parameter family of Riemannian metrics $g(t), t \in[0, T]$, evolving by

$$
\frac{\partial g(t, x)}{\partial t}=-2 \alpha(t, x)
$$

Date: August 21, 2018.

2010 Mathematics Subject Classification. Primary 53C44. 
Here $\alpha(t, x)$ is a one-parameter family of smooth symmetric 2-tensors on $M$. In particular, when $\alpha=\mathrm{Rc}$, Eq.(1.1) is R. Hamilton's Ricci flow. Let

$$
\mathrm{S}(t, x) \doteqdot g^{i j} \alpha_{i j}
$$

be the trace of $\alpha$ with respect to the time-dependent metric $g(t)$.

In [7], R. Müller studied reduced volumes for the abstract flow (1.1) and defined the following quantity for tensor $\alpha$ and vector $V$,

$$
\begin{aligned}
\mathcal{D}_{\alpha}(V) \doteqdot & \frac{\partial \mathrm{S}}{\partial t}-\Delta \mathrm{S}-2|\alpha|^{2} \\
& +2(\mathrm{Rc}-\alpha)(V, V)+\langle 4 \operatorname{Div}(\alpha)-2 \nabla \mathrm{S}, V\rangle
\end{aligned}
$$

where Div is the divergence operator defined by $\operatorname{Div}(\alpha)_{k}=g^{i j} \nabla_{i} \alpha_{j k}$ (in local coordinates). Under the assumption that $\mathcal{D}_{\alpha} \geq 0$, Müller obtained monotonicity of the reduced volumes [7]. Most recently, in [6], the authors proved monotonicity for the entropy and the lowest eigenvalue. In [5], a Harnack inequality for positive solutions of the conjugate heat equation and heat equation with potential has been proved.

The main purpose of this article is to derive Harnack inequalities for a conjugate heat kernel in the abstract setting with $\mathcal{D}_{\alpha} \geq 0$.

1.1. Main Results. We consider $(M, g(t)), 0 \leq t \leq T$, to be a solution of (1.1) and $\tau \doteqdot T-t$,

$$
\square^{*} \doteqdot-\frac{\partial}{\partial t}-\Delta+\mathrm{S}=\frac{\partial}{\partial \tau}-\Delta+\mathrm{S}
$$

A function $u=(4 \pi \tau)^{-n / 2} e^{-f}$ is a solution to the conjugate heat equation if,

$$
\square^{*} u=0
$$

We also denote

$$
H(x, t ; y, T)=(4 \pi(T-t))^{-n / 2} e^{-h}=(4 \pi \tau)^{-n / 2} e^{-h}
$$

to be a heat kernel. That is, based at a fixed $(x, t), \mathrm{H}$ is the fundamental solution of heat equation $\square H=0$, and similarly for fixed $(y, T)$ and conjugate heat equation $\square^{*} H=0$. Our first result is computational.

Theorem 1.1. Let

$$
v=\left(\tau\left(2 \Delta f-|\nabla f|^{2}+\mathrm{S}\right)+f-n\right) u
$$


then we have

$$
\square^{*} v=-2 \tau u\left|\alpha+\nabla \nabla f-\frac{g}{2 \tau}\right|^{2}-\tau u \mathcal{D}_{\alpha}(\nabla f) .
$$

Secondly, we obtain the following Harnack estimate.

Theorem 1.2. If $\mathcal{D}_{\alpha} \geq 0$, then the following inequality holds,

$$
\tau\left(2 \Delta h-|\nabla h|^{2}+\mathrm{S}\right)+h-n \leq 0 .
$$

Remark 1.3. For the Ricci flow, where $\alpha=\operatorname{Rc}$, one has $\mathcal{D}=0$; (1.5) has been proved by G. Perelman [10]. On a static Riemannian manifold where $\alpha=0$ one has $\mathcal{D}=\mathrm{Rc}$, and (1.5) has been proved by L. Ni in [8] for static manifolds with nonnegative Ricci curvature. Another special case of (1.5) was recently proved by the third author for the extended Ricci flow in [12]. (1.5) is new for Müller's RicciHarmonic flow and mean curvature flow in a Lorentzian manifold with nonnegative sectional curvature. The detailed calculations of $\mathcal{D}$ can be found in [7].

Acknowledgement. X. Cao was partially supported by a grant from the Simons Foundation (\#280161) and by the Jeffrey Sean Lehman Fund from Cornell University; H. Guo was supported by NSF of China (Grant No. 11171143) and Zhejiang Provincial Natural Science Foundation of China (Project No. LY13A010009).

\section{Preliminaries}

2.1. Evolution Equations. In this section, we collect several evolution equations and prove Theorem 1.1 .

For the Laplace-Beltrami operator $\Delta$ with respect to $g(t)$ we have,

$$
\left(\frac{\partial}{\partial t} \Delta\right) f=2\langle\alpha, \nabla \nabla f\rangle+\langle 2 \operatorname{Div}(\alpha)-\nabla \mathrm{S}, \nabla f\rangle
$$

where $f$ is any smooth function on $M$. This formula can be found in standard textbooks, for instance [3].

Now we assume $u$ is a solution to the conjugate heat equation. The operator $-\square^{*}$ acting on the term $u \log u$ produces,

$$
-\square^{*} u \log u=u|\nabla \log u|^{2}+u \mathrm{~S} .
$$

The same operator acts once more and we have,

$$
\begin{aligned}
& -\square^{*}\left(u|\nabla \log u|^{2}+u \mathrm{~S}\right) \\
& =2 u \alpha(\nabla \log u, \nabla \log u)+4\langle\nabla \mathrm{S}, \nabla u\rangle+u \frac{\partial \mathrm{S}}{\partial t}+2 u|\nabla \nabla \log u|^{2}
\end{aligned}
$$




$$
\begin{aligned}
& +2 u \operatorname{Rc}(\nabla \log u, \nabla \log u)+u \Delta \mathrm{S} \\
& =2 u|\nabla \nabla \log u-\alpha|^{2}+4 u\langle\alpha, \nabla \nabla \log u\rangle+2 u \alpha(\nabla \log u, \nabla \log u) \\
& -2 u|\alpha|^{2}+4\langle\nabla \mathrm{S}, \nabla u\rangle+u \frac{\partial \mathrm{S}}{\partial t}+2 u \operatorname{Rc}(\nabla \log u, \nabla \log u)+u \Delta \mathrm{S} .
\end{aligned}
$$

Notice that,

$-\square *(\Delta u)=2\langle\alpha, \nabla \nabla u\rangle+\langle 2 \operatorname{Div}(\alpha)-\nabla \mathrm{S}, \nabla u\rangle+2\langle\nabla \mathrm{S}, \nabla u\rangle+u \Delta \mathrm{S}$

$$
\begin{aligned}
= & 2 u\langle\alpha, \nabla \nabla \log u\rangle+2 u \alpha(\nabla \log u, \nabla \log u) \\
& +\langle 2 \operatorname{Div}(\alpha)-\nabla \mathrm{S}, \nabla u\rangle+2\langle\nabla \mathrm{S}, \nabla u\rangle+u \Delta \mathrm{S} .
\end{aligned}
$$

Thus, by (1.2), we have, for $V=-\nabla \log u$,

$$
-\square^{*}\left(u|\nabla \log u|^{2}+u \mathrm{~S}-2 \Delta u\right)=2 u|\nabla \nabla \log u-\alpha|^{2}+u \mathcal{D}_{\alpha}(V)
$$

Moreover,

$$
\begin{aligned}
& -\square^{*}\left(\tau\left(u|\nabla \log u|^{2}+u S-2 \Delta u\right)-u \log u-\frac{n u}{2} \log \tau\right) \\
& =2 \tau u\left|\alpha-\nabla \nabla \log u-\frac{g}{2 \tau}\right|^{2}+\tau u \mathcal{D}_{\alpha}(-\nabla \log u) .
\end{aligned}
$$

In the calculation above, if we add a normalization term $c_{n} u$ to the left hand side, we get the same on the right hand side since $\square^{*} u=0$. Thus, we have the following result.

\section{Lemma 2.1.}

$$
\begin{aligned}
& \square^{*}\left[\tau\left(u|\nabla \log u|^{2}+u \mathrm{~S}-2 \Delta u\right)-u \log u-\frac{n u}{2} \log (4 \pi \tau)-n u\right] \\
& =-2 \tau u\left|\alpha-\nabla \nabla \log u-\frac{g}{2 \tau}\right|^{2}-\tau u \mathcal{D}_{\alpha}(-\nabla \log u) .
\end{aligned}
$$

Theorem 1.1 follows by realizing that $f \doteqdot-\log u-\frac{n}{2} \log (4 \pi \tau)$.

2.2. Asymptotic Behavior and Reduced Geometry. Let's recall the asymptotic behavior of the heat kernel as $t \rightarrow T$.

Theorem 2.2. [1, Theorem 24.21] For $\tau=T-t$,

$$
H(x, t ; y, T) \sim \frac{e^{-\frac{d_{T}^{2}(x, y)}{4 \tau}}}{(4 \pi \tau)^{n / 2}} \Sigma_{j=0}^{\infty} \tau^{j} u_{j}(x, y, \tau) .
$$

More precisely, there exist $t_{0}>0$ and a sequence $u_{j} \in C^{\infty}(M \times M \times$ $\left.\left[0, t_{0}\right]\right)$ such that,

$$
H(x, t ; y, T)-\frac{e^{-\frac{d_{T}^{2}(x, y)}{4 \tau}}}{(4 \pi \tau)^{n / 2}} \Sigma_{j=0}^{k} \tau^{j} u_{j}(x, y, T-l)=w_{k}(x, y, \tau),
$$


with

$$
\begin{aligned}
u_{0}(x, x, 0) & =1, \\
w_{k}(x, y, \tau) & =O\left(\tau^{k+1-\frac{n}{2}}\right),
\end{aligned}
$$

as $\tau \rightarrow 0$ uniformly for all $x, y \in M$.

Then following [7], we can define reduced length and distance.

Definition 2.3. Given $\tau(t)=T-t$, we define the $\mathcal{L}$-length of a curve $\gamma:\left[\tau_{0}, \tau_{1}\right] \mapsto N,\left[\tau_{0}, \tau_{1}\right] \subset[0, T]$ by,

$$
\mathcal{L}(\gamma):=\int_{\tau_{0}}^{\tau_{1}} \sqrt{\tau}\left(\mathrm{S}(\gamma(\tau))+|\dot{\gamma}(\tau)|^{2}\right) d \tau
$$

For a fixed point $y \in N$ and $\tau_{0}=0$, the backward reduced distance is defined as,

$$
\ell\left(x, \tau_{1}\right):=\inf _{\gamma \in \Gamma}\left\{\frac{1}{2 \tau_{1}} \mathcal{L}(\gamma)\right\}
$$

where $\Gamma=\left\{\gamma:\left[0, \tau_{1}\right] \mapsto M, \gamma(0)=y, \gamma\left(\tau_{1}\right)=x\right\}$.

The backward reduced volume is defined as

$$
V(\tau):=\int_{M}(4 \pi \tau)^{-n / 2} e^{-\ell(y, \tau)} d \mu_{\tau}(y) .
$$

The next result, mainly from [7], relates the reduced distance defined in (2.14) with the distance at time $\mathrm{T}$.

Lemma 2.4. Let $L(x, \tau)=4 \tau \ell(x, \tau)$ then we have the followings:

a. Assume that there exists $k_{1}, k_{2} \geq 0$ such that $-k_{1} g(t) \leq \alpha(t) \leq$ $k_{2} g(t)$ for $t \in[0, T]$, then $L$ is smooth amost everywhere and a local Lipschitz function on $N \times[0, T]$. Furthermore,

$$
e^{-2 k_{1} \tau} d_{T}^{2}(x, y)-\frac{4 k_{1} n}{3} \tau^{2} \leq L(x, \tau) \leq e^{2 k_{2} \tau} d_{T}^{2}(x, y)+\frac{4 k_{2} n}{3} \tau^{2} .
$$

b. If $\mathcal{D}_{\alpha} \geq 0$, then $\square^{*}\left(\frac{e^{-\frac{L(x, \tau)}{4 \tau}}}{(4 \pi \tau)^{n / 2}}\right) \leq 0$.

c. For the same point $y$ in the definition of reduced distance and $H(x, t ; y, T)=(4 \pi \tau)^{-n / 2} e^{-h}$, then $h(x, t ; y, T) \leq \ell(x, T-t)$.

Proof. Parts a. and b. follow from [7, Lemmas 4.1, 5.15] respectively.

For part c. we provide a brief argument here (for more details, see [2, Lemma 16.49]).

We first observe that part a. implies $\lim _{\tau \rightarrow 0} L(x, \tau)=d_{T}^{2}(y, x)$ and,

$$
\lim _{\tau \rightarrow 0} \frac{e^{-\frac{L_{w}(x, \tau)}{4 \tau}}}{(4 \pi \tau)^{n / 2}}=\delta_{y}(x),
$$


since Riemannian manifolds locally look like Euclidean. It then follows from part $\mathbf{b}$. and maximum principle that,

$$
H(x, t ; y, T) \geq \frac{e^{-\frac{L(x, \tau)}{4 \tau}}}{(4 \pi \tau)^{n / 2}}=\frac{e^{-\frac{L(x, T-t)}{4 \tau}}}{(4 \pi(T-t))^{n / 2}} .
$$

Hence,

$$
h(x, t ; y, T) \leq \frac{L(x, \tau)}{4 \tau}=\ell(x, \tau)=\ell(x, T-l)
$$

2.3. Entropy Formulas. In this subsection, we define several functionals and collect their properties.

Definition 2.5. Along flow (1.1), for h satisfying $\int_{M}(4 \pi \tau)^{-n / 2} e^{-h} d \mu=$ 1, we define

$$
\mathbb{W}_{\alpha}(g, \tau, h) \doteqdot \int_{M}\left(\tau\left(|\nabla h|^{2}+\mathrm{S}\right)+(h-n)\right)(4 \pi \tau)^{-n / 2} e^{-h} d \mu
$$

Associated functionals are defined as follows:

$$
\begin{aligned}
\mu_{\alpha}(g, \tau) & =\inf _{f} \mathbb{W}_{\alpha}(g, h, \tau), \\
v_{\alpha}(g) & =\inf _{\tau>0} \mu_{\alpha}(g, \tau) .
\end{aligned}
$$

Remark 2.6. Since $\alpha$ is a (2,0)-tensor, S scales like the inverse of the metric. Thus, these functionals satisfy diffeomorphism invariance and the following scaling rules:

$$
\begin{aligned}
\mathbb{W}_{\alpha}(g, \tau, h) & =\mathbb{W}_{\alpha}(c g, c \tau, h), \\
\mu_{\alpha}(g, \tau) & =\mu_{\alpha}(c g, c \tau), \\
v_{\alpha}(g, u) & =v_{\alpha}(c g) .
\end{aligned}
$$

Next, we collect some useful results.

Lemma 2.7. On a closed Riemannian manifold $(M, g(t)), t \in[0, T]$, evolved by (1.1), with $\mathcal{D}_{\alpha} \geq 0$. Let $\tau=T-t$, the following holds:

a. $\mathbb{W}_{\alpha}(g, \tau, h)$ is non-decreasing in time $t$ (non-increasing in $\tau$ ).

b. There exists a smooth minimizer $h_{\tau}$ for $\mathbb{W}_{\alpha}(g, \tau,$.$) which satisfies$

$$
\tau\left(2 \triangle h_{\tau}-\left|\nabla h_{\tau}\right|^{2}+\mathrm{S}\right)+h_{\tau}-n=\mu_{\alpha}(g, \tau) .
$$

In particular, $\mu_{\alpha}(g, \tau)$ is finite.

c. $\mu_{\alpha}(g, \tau)$ is non-decreasing in time $t$.

d. $\lim _{\tau \rightarrow 0^{+}} \mu_{\alpha}(g, \tau)=0$. 
Proof. Part a. follows from [6, Theorem 5.2].

Part b. is deducted from the regularity theory for elliptic equations based on Sobolev spaces. The details can be found in [1, Proposition 17.24]. Replacing $\mathrm{R}$ by $\mathrm{S}$, the argument works exactly the same.

Part c. is an immediate consequence of the monotonicity formula (part a.) and the existence of a minimizer realizing the $\mu_{\alpha}$ functional (part b.).

The proof of part $\mathbf{d}$. is mostly identical to that of [11, Prop 3.2] (also [1, Prop 17.19, 17.20]), but it is subtle so we give a brief argument here.

Assume that the flow exists for $\tau \in[0, \bar{\tau}]$. The idea is to construct cut-off functions reflecting the local geometry which looks like Euclidean. Then it is shown that the limit of $\mathbb{W}_{\alpha}$ functional on these functions is 0 if a certain parameter approaches 0 . Thus, by the monotonicity of $\mu_{\alpha}$ and L. Gross's logarithmic-Sobolev inequality on an Euclidean space [4], the result then follows.

The construction of cut-off functions follows [11, Prop 3.2]. Let $\tau_{0}=\bar{\tau}-\epsilon$ for small $\epsilon$. Using normal coordinates at a point $\mathrm{p}$ on $\left(M, g\left(\tau_{0}\right)\right)$, we define a cut-off function

$$
f_{1}= \begin{cases}\frac{|x|^{2}}{4 \epsilon} & \text { if } d(x, p)=|x|<\rho, \\ \frac{\rho^{2}}{4 \epsilon} & \text { elsewhere, }\end{cases}
$$

where $\rho$ is a positive number smaller than the injectivity radius (which exists since $M$ is closed). Then by the choice of our coordinate,

$$
d \mu\left(\tau_{0}\right)=1+O\left(|x|^{2}\right),|x|<<1
$$

and let

$$
e^{-C} \doteqdot \int_{M}(4 \pi \epsilon)^{-n / 2} e^{f_{1}}
$$

then $C \rightarrow 0$ as $\epsilon \rightarrow 0$.

Let $f=f_{1}+C$ then,

$$
\begin{aligned}
u & \doteqdot(4 \pi \epsilon)^{-n / 2} e^{-f} \\
1 & =\int_{M}(4 \pi \epsilon)^{-n / 2} e^{-f} d \mu\left(\tau_{0}\right) ; \\
|\nabla f|^{2} & =\left|\nabla f_{1}\right|^{2}=\left|\nabla \frac{|x|^{2}}{4 \epsilon}\right|^{2}=\frac{|x|^{2}}{4 \epsilon}, \text { for }|x|<\rho .
\end{aligned}
$$

We solve $\mathrm{f}$ backward using equation

$$
\frac{\partial}{\partial t} f=-\mathrm{S}-\Delta f+|\nabla f|^{2}+\frac{n}{2 \tau} .
$$


The solution clearly depends on the choice of $\epsilon$. Now using (2.16), we calculate,

$$
\begin{aligned}
\mathbb{W}\left(g\left(\tau_{0}\right), \bar{\tau}-\tau_{0}, f\left(\tau_{0}\right)\right)= & \int_{|x|<\rho}\left(\epsilon\left(\mathrm{S}+\frac{|x|^{2}}{4 \epsilon^{2}}\right)+\frac{|x|^{2}}{4 \epsilon^{2}}+C-n\right) u d \mu \\
& +\int_{d(x, p) \geq \rho}\left(\epsilon \mathrm{S}+\frac{\rho^{2}}{4 \epsilon}+C-n\right) u d \mu \\
& =\int_{|x|<\rho}\left(\frac{|x|^{2}}{2 \epsilon}-n\right) u d \mu+\epsilon \int_{M} \mathrm{~S} u d \mu \\
& +C \int_{M} u d \mu+\int_{d(x, p) \geq \rho}\left(\frac{\rho^{2}}{4 \epsilon}-n\right) u d \mu \\
& =I+I I+I I I+I V .
\end{aligned}
$$

By a change of variable, as $\epsilon \rightarrow 0$, we have,

$$
\begin{aligned}
I I+I I I & \rightarrow 0 ; \\
I V & =\int_{d(x, p) \geq \rho}\left(\frac{\rho^{2}}{4 \epsilon}-n\right) e^{-\frac{\rho^{2}}{4 \epsilon}-C} \rightarrow 0 ; \\
I & =e^{-C} \int_{|y| \leq \frac{\rho}{\sqrt{\epsilon}}}\left(\frac{|y|^{2}}{2}-n\right)(2 \pi)^{-n / 2} e^{-|y|^{2} / 4}\left(1+O\left(\epsilon|y|^{2}\right) d y\right. \\
& \rightarrow \int_{\mathbb{R}^{n}}\left(\frac{|y|^{2}}{2}-n\right)(2 \pi)^{-n / 2} e^{-|y|^{2} / 4} d y=0 .
\end{aligned}
$$

Thus, by part $\mathbf{a}$. and $\mathbf{b}, \mu_{\alpha}(g(t), \bar{\tau}-t) \leq 0$ for any $t \leq \bar{\tau}$. The proof that the limit is actually 0 when $\tau \rightarrow 0^{+}$follows from a rather standard blow-up argument whose details can be found in either [11, Prop 3.2] or [1, Prop 17.20].

\section{Estimates on the Heat Kernel}

In this section, we obtain several estimates on the heat kernel using maximum principle and the monotone framework. Particularly, we derive a gradient estimate and an upper bound for positive solutions of the conjugate heat equation. Then we prove our main result.

3.1. A Gradient Estimate. We first establish a space-only gradient estimate. Recall that,

$$
\square^{*}=\frac{\partial}{\partial \tau}-\Delta+\mathrm{S}
$$


Lemma 3.1. Assume there exist $k_{1}, k_{2}, k_{3}, k_{4}>0$ such that the followings hold on $N \times[0, T]$,

$$
\begin{aligned}
\operatorname{Rc}(g(t)) & \geq-k_{1} g(t), \\
\alpha & \geq-k_{2} g(t), \\
|\nabla \mathrm{S}|^{2} & \leq k_{3}, \\
|\mathrm{~S}| & \leq k_{4} .
\end{aligned}
$$

Let $q$ be any positive solution to the conjugate heat equation on $M \times$ $[0, T]$, i.e., $\square^{*} q=0$, and $\tau=T-t$. If $q<Q$ for some constant $Q$ then there exist $C_{1}, C_{2}$ depending on $k_{1}, k_{2}, k_{3}, k_{4}$ and $n$, such that for $0<\tau \leq \min \{1, T\}$, we have

$$
\tau \frac{|\nabla q|^{2}}{q^{2}} \leq\left(1+C_{1} \tau\right)\left(\ln \frac{Q}{q}+C_{2} \tau\right)
$$

Proof. We compute that

$$
\begin{aligned}
\left(-\frac{\partial}{\partial t}-\triangle\right) \frac{|\nabla q|^{2}}{q}= & \mathrm{S} \frac{|\nabla q|^{2}}{q}+\frac{1}{q}\left(-\frac{\partial}{\partial t}-\triangle\right)|\nabla q|^{2}+2|\nabla q|^{2} \nabla \frac{1}{q} \nabla \ln q \\
& -2 \nabla|\nabla q|^{2} \nabla \frac{1}{q}, \\
\frac{1}{q}\left(-\frac{\partial}{\partial t}-\triangle\right)|\nabla q|^{2}= & \frac{1}{q}\left[-2(\alpha+\mathrm{Rc})(\nabla q, \nabla q)-2 \nabla q \nabla(\mathrm{S} q)-2\left|\nabla^{2} q\right|^{2}\right], \\
2|\nabla q|^{2} \nabla \frac{1}{q} \nabla \ln q & =-2 \frac{|\nabla q|^{4}}{q^{3}}, \\
-2 \nabla|\nabla q|^{2} \nabla \frac{1}{q} & =\frac{\nabla^{2} q(\nabla q, \nabla q)}{q^{2}} .
\end{aligned}
$$

Thus

$$
\begin{aligned}
\left(-\frac{\partial}{\partial t}-\triangle\right) \frac{|\nabla q|^{2}}{q}= & \frac{-2}{q}\left|\nabla^{2} q-\frac{d q \otimes d q}{q}\right|^{2}+\mathrm{S} \frac{|\nabla q|^{2}}{q} \\
& +\frac{-2(\alpha+\mathrm{Rc})(\nabla q, \nabla q)-2 \mathrm{~S} \nabla q \nabla q-2 q \nabla q \nabla \mathrm{S}}{q} \\
\leq & {\left[2\left(k_{1}+k_{2}\right)+n k_{2}\right] \frac{|\nabla q|^{2}}{q}+2|\nabla q||\nabla \mathrm{S}| } \\
\leq & {\left[2 k_{1}+(2+n) k_{2}+1\right] \frac{|\nabla q|^{2}}{q}+k_{3} q . }
\end{aligned}
$$

Furthermore, we have

$$
\left(-\frac{\partial}{\partial t}-\triangle\right)\left(q \ln \frac{Q}{q}\right)=-\mathrm{S} q \ln \frac{Q}{q}+\mathrm{S} q+\frac{|\nabla q|^{2}}{q}
$$




$$
\geq \frac{|\nabla q|^{2}}{q}-n k_{2} q-k_{4} q \ln \frac{Q}{q}
$$

Let $\Phi=a(\tau) \frac{|\nabla q|^{2}}{q}-b(\tau) q \ln \frac{Q}{q}-c(\tau) q$, then

$$
\begin{aligned}
\left(-\frac{\partial}{\partial t}-\triangle\right) \Phi \leq & \frac{|\nabla q|^{2}}{q}\left(a^{\prime}(\tau)+a(\tau)\left(2 k_{1}+(2+n) k_{2}+1\right)-b(\tau)\right) \\
& +q \ln \frac{Q}{q}\left(k_{4} b(\tau)-b^{\prime}(\tau)\right) \\
& +q\left(k_{3} a(\tau)-c^{\prime}(\tau)+n k_{2} b(\tau)+c(\tau) k_{4}\right) .
\end{aligned}
$$

We can now choose a, b and c appropriately such that $\left(-\partial_{t}-\triangle\right) \Phi \leq 0$. For example, take

$$
\begin{aligned}
a & =\frac{\tau}{1+\left(2 k_{1}+(2+n) k_{2}+1\right) \tau} \\
b & =e^{k_{4} \tau} \\
c & =\left(e^{k_{5} k_{4} \tau} n k_{2}+k_{3}\right) \tau,
\end{aligned}
$$

for $k_{5}=1+\frac{k_{3}}{n k_{2}}$. Then by maximum principle, noticing that $\Phi \leq 0$ at $\tau=0$, we arrive at

$$
a \frac{|\nabla q|^{2}}{q} \leq b(\tau) q \ln \frac{Q}{q}+c q .
$$

The result then follows from simple algebra.

3.2. $L_{\infty}$ Bound. Second, we shall derive an upper bound for positive conjugate heat solutions. Our main statement says that any normalized solution can not blow up too fast.

Lemma 3.2. Let $q$ be any normalized positive solution to the conjugate heat equation on $M \times[0, T]$, i.e., $\square^{*} q=0$ with $\int q d \mu_{g(0)}=1$. Let $\tau=T-t$, then there exists a constant $C$ depending on the geometry of $g(t)_{t \in[0, T]}$, such that

$$
q(y, \tau) \leq \frac{C}{\tau^{n / 2}} .
$$

Proof. The proof is modeled after [9, Lemma 2.2] (also see [2, Lemma 16.47]). As the solution and the flow is well defined in $M \times[0, T]$, there exists $y_{0}, \tau_{0}$ such that

$$
\sup _{M \times[0, \min \{1, T\}]} \tau^{n / 2} q(y, \tau)=\tau_{0}^{n / 2} q\left(y_{0}, \tau_{0}\right) .
$$


In particular,

$$
\sup _{M \times\left[\tau_{0} / 2, \tau_{0}\right]} q(y, \tau) \leq \frac{\tau_{0}^{n / 2}}{\tau^{n / 2}} q\left(y_{0}, \tau_{0}\right) \leq 2^{n / 2} q\left(y_{0}, \tau_{0}\right):=Q .
$$

Applying Lemma 3.1 to $q(y, \tau)$ on $M \times\left[\tau_{0} / 2, \tau_{0}\right]$ we obtain,

$$
\frac{\tau_{0}}{2} \frac{|\nabla q|^{2}}{q^{2}}\left(y, \tau_{0}\right) \leq\left(1+C_{1} \frac{\tau_{0}}{2}\right)\left(\log \left(\frac{Q}{q\left(y, \tau_{0}\right)}\right)+C_{2} \frac{\tau_{0}}{2}\right)
$$

Let $G\left(y, \tau_{0}\right):=\log \left(\frac{Q}{q\left(y, \tau_{0}\right)}\right)+C_{2} \frac{\tau_{0}}{2}$, then the inequality above can be rewritten as

$$
|\nabla \sqrt{G}|^{2}=\left|\frac{1}{2} \frac{\nabla G}{G^{1 / 2}}\right|^{2}=\left|\frac{1}{2 G^{1 / 2}} \frac{\nabla q}{q}\right|^{2}=\frac{1}{4 G} \frac{|\nabla q|^{2}}{q^{2}} \leq \frac{1+C_{1} \frac{\tau_{0}}{2}}{2 \tau_{0}} .
$$

Therefore, with $B_{\tau}(y, r)$ denoting the ball of radius r measured by $g(\tau)$ around the point $\mathrm{y}$, we have

$\sup _{B_{\tau_{0}}\left(y_{0}, \sqrt{\frac{\tau_{0}}{1+C_{1} \frac{\tau_{0}}{2}}}\right)} \sqrt{G}\left(y, \tau_{0}\right) \leq \sqrt{G}\left(y_{0}, \tau_{0}\right)+\frac{1}{\sqrt{2}}=\sqrt{\frac{n}{2} \log 2+C_{2} \frac{\tau_{0}}{2}}+\frac{1}{\sqrt{2}}$.

Writing the above inequality in terms of $q\left(y, \tau_{0}\right)$ yields,

$q\left(y, \tau_{0}\right) \geq q\left(y_{0}, \tau_{0}\right) \exp \left\{-\frac{1}{2}-\frac{n}{2} \log 2-\frac{2}{\sqrt{2}} \sqrt{\frac{n}{2} \log 2+\frac{C_{2}}{2}}\right\}:=C_{3} q\left(y_{0}, \tau_{0}\right)$.

Now we observe that there exists a constant $C_{4}$ depending on the geometry of $\left(M, g\left(\tau_{0}\right)\right)$, such that

$$
\operatorname{Vol}_{g\left(\tau_{0}\right)}\left(B_{\tau_{0}}\left(y_{0}, \sqrt{\frac{\tau_{0}}{1+C_{1} \frac{\tau_{0}}{2}}}\right) \geq C_{4} \tau_{0}^{n / 2} .\right.
$$

Therefore we have,

$$
q\left(y_{0}, \tau_{0}\right) \leq \frac{1}{C_{3} C_{4} \tau_{0}^{n / 2}} \int_{M} q\left(y, \tau_{0}\right) d \mu_{\tau_{0}}(y):=\frac{C_{5}}{\tau_{0}^{n / 2}} \int_{M} q\left(y, \tau_{0}\right) d \mu_{\tau_{0}}(y) .
$$

By our choice of $y_{0}, \tau_{0}$ and the fact that $\int_{M} q(y, \tau) d \mu_{\tau}(y)$ remains constant along the flow, the statement follows.

Remark 3.3. It is interesting to note that in [13], a Harnack inequality is used to obtain an off-diagonal bound, while here the argument goes the opposite direction. 
3.3. Proofs of Main Results. Finally, we are ready to finish our proof of the main theorem.

Lemma 3.4. Let $H(x, t ; y, T)=(4 \pi \tau)^{-n / 2} e^{-h}$ be a heat kernel and $\Phi$ be any positive solution to the heat equation. Then we have

$$
\int_{M} h H \Phi d \mu \leq \frac{n}{2} \Phi(y, T), \text { i.e, } \quad \int_{M}\left(h-\frac{n}{2}\right) H \Phi d \mu \leq 0 .
$$

Proof. By Lemma 2.4 we have

$$
\begin{array}{r}
\limsup _{\tau \rightarrow 0} \int_{M} h H \Phi d \mu \leq \limsup _{\tau \rightarrow 0} \int_{M} \ell(x, \tau) H \Phi d \mu(x) \\
\leq \limsup _{\tau \rightarrow 0} \int_{M} \frac{d_{T}^{2}(x, y)}{4 \tau} H \Phi d \mu(x) .
\end{array}
$$

Using Theorem 2.2, it follows that,

$$
\lim _{\tau \rightarrow 0} \int_{M} \frac{d_{T}^{2}(x, y)}{4 \tau} H \Phi d \mu(x)=\lim _{\tau \rightarrow 0} \int_{M} \frac{d_{T}^{2}(x, y)}{4 \tau} \frac{e^{-\frac{d_{T}^{2}(x, y)}{4 \tau}}}{(4 \pi \tau)^{n / 2}} \Phi d \mu(x) .
$$

Either by differentiating twice under the integral sign or using these following identities on Euclidean spaces

$$
\int_{-\infty}^{\infty} e^{-a \mathbf{x}^{2}} d \mathbf{x}=\sqrt{\frac{\pi}{a}} \text { and } \int_{-\infty}^{\infty} \mathbf{x}^{2} e^{-a \mathbf{x}^{2}} d \mathbf{x}=\frac{1}{2 a} \sqrt{\frac{\pi}{a}},
$$

we obtain that

$$
\int_{\mathrm{R}^{n}}|x|^{2} e^{-a|x|^{2}} d x=n\left(\int_{-\infty}^{\infty} \mathbf{x}^{2} e^{-a \mathbf{x}^{2}} d \mathbf{x}\right)\left(\int_{-\infty}^{\infty} e^{-a \mathbf{x}^{2}} d \mathbf{x}\right)^{n-1}=\frac{n}{2 a}\left(\frac{\pi}{a}\right)^{n / 2} .
$$

Therefore,

$$
\lim _{\tau \rightarrow 0} \frac{d_{T}^{2}(x, y)}{4 \tau} \frac{e^{-\frac{d_{T}^{2}(x, y)}{4 \tau}}}{(4 \pi \tau)^{n / 2}}=\frac{n}{2} \delta_{y}(x),
$$

hence

$$
\lim _{\tau \rightarrow 0} \int \frac{d_{T}^{2}(x, y)}{4 \tau} \frac{e^{-\frac{d_{T}^{2}(x, y)}{4 \tau}}}{(4 \pi \tau)^{n / 2}} \Phi d \mu_{N}(x)=\frac{n}{2} \Phi(y, T) .
$$

Thus the result follows.

The following result implies that the equality actually holds.

Proposition 3.5. Let $H(x, t ; y, T)=(4 \pi \tau)^{-n / 2} e^{-h}$ be a heat kernel and $\Phi$ be any positive solution to the heat equation. Then for

$$
\begin{aligned}
v & =\left[(T-t)\left(2 \triangle h-|\nabla h|^{2}+S\right)+h-n\right] H, \\
\rho_{\Phi}(t) & =\int_{M} v \Phi d \mu,
\end{aligned}
$$


we have

$$
\lim _{t \rightarrow T} \rho_{\Phi}(t)=0 .
$$

Proof. Integrating by parts yields that

$$
\begin{aligned}
\rho_{\Phi}(t)= & \int_{M}\left[\tau\left(2 \triangle h-|\nabla h|^{2}+S\right)+h-n\right] H \Phi d \mu \\
& =-\int_{M} 2 \tau \nabla h \nabla(H \Phi) d \mu-\int_{M} \tau|\nabla h|^{2} H \Phi d \mu+\int_{M}(\tau \mathrm{S}+h-n) H \Phi d \mu \\
& =\int_{M} \tau|\nabla h|^{2} H \Phi d \mu-2 \tau \int_{M} \nabla \Phi \nabla h H d \mu+\int_{M}(\tau \mathrm{S}+h-n) H \Phi d \mu \\
& =\int_{M} \tau|\nabla h|^{2} H \Phi d \mu-2 \tau \int_{M} H \triangle \Phi d \mu+\int_{M}(\tau \mathrm{S}+h-n) H \Phi d \mu \\
= & \int_{M} \tau|\nabla h|^{2} H \Phi d \mu+\int_{M} h H \Phi d \mu-2 \tau \int_{M} H \triangle \Phi d \mu+\int_{M}(\tau \mathrm{S}-n) H \Phi d \mu .
\end{aligned}
$$

Notice that, except the first two terms, the rest approaches $-n \Phi(y, T)$ as $\tau \rightarrow 0$. For the first term, using Lemmas 3.2 and 3.1 for any space$(\tau)$ time point on $M \times\left[\frac{\tau}{2}, \tau\right]$ we arrive at

$$
\begin{aligned}
\tau \int_{M}|\nabla h|^{2} H \Phi d \mu & \leq\left(2+C_{1} \tau\right) \int_{M}\left(\ln \left(\frac{C_{3}}{H \tau^{n / 2}}\right)+C_{2} \tau\right) H \Phi d \mu \\
& \leq\left(2+C_{1} \tau\right) \int_{M}\left(\ln C_{3}+h+\frac{n}{2} \ln (4 \pi)+C_{2} \tau\right) H \Phi d \mu,
\end{aligned}
$$

with $C_{1}, C_{2}$ defined as in Lemma 3.1 , while $C_{3}$ is a constant depending on the geometry of $g(t), \frac{\tau}{2} \leq T-t \leq \tau$. As $\tau \rightarrow 0, \ln C_{3}+\frac{n}{2} \ln (4 \pi)$ is bounded from above by another constant $C_{4}$ also depending on the geometry of $g(t), t \in[0, T]$. Consequently, by Lemma 3.4, which claims the finiteness of $\int_{M} h H \Phi d \mu$,

$$
\begin{aligned}
\lim _{\tau \rightarrow 0}\left(\int_{M} \tau|\nabla h|^{2} d \mu+\int_{M} h H \Phi d \mu\right) & \leq 3 \int_{M} h H \Phi d \mu+2 \ln C_{4} \Phi(x, T) \\
& \leq\left(\frac{3 n}{2}+2 \ln C_{4}\right) \Phi(x, T) .
\end{aligned}
$$

Thus we have

$$
\lim _{t \rightarrow T} \rho_{\Phi}(t) \leq C_{5} \Phi(x, T) .
$$

Since $\Phi$ is a positive solution satisfying $\partial_{t} \Phi=\triangle \Phi$, applying Theorem 1.1 yields that,

$$
\partial_{t} \rho_{\Phi}(t)=\partial_{t} \int v \Phi d \mu=\int\left(\square \Phi v-\Phi \square^{*} v\right) d \mu \geq 0
$$


The above implies that there exists $\beta$, such that

$$
\lim _{t \rightarrow T} \rho_{\Phi}(t)=\beta \text {. }
$$

Hence

$$
\lim _{\tau \rightarrow 0}\left(\rho_{\Phi}(T-\tau)-\rho_{\Phi}\left(T-\frac{\tau}{2}\right)\right)=0 .
$$

By the above equation (3.22), Theorem 1.1, and the mean-value theorem, there exists a sequence $\tau_{i} \rightarrow 0$, such that

$$
\lim _{\tau_{i} \rightarrow 0} \tau_{i}^{2} \int_{M}\left(\left|\alpha+\operatorname{Hess} h-\frac{g}{2 \tau_{i}}\right|^{2}+\frac{1}{2} \mathcal{D}_{\alpha}(\nabla h)\right) H \Phi d \mu=0 .
$$

Now standard inequalities yield that,

$$
\begin{aligned}
& {\left[\int_{M} \tau_{i}\left(\mathrm{~S}+\Delta h-\frac{n}{2 \tau_{i}}\right) H \Phi d \mu\right]^{2} } \\
\leq & {\left[\int_{M} \tau_{i}^{2}\left(S+\triangle h-\frac{n}{2 \tau_{i}}\right)^{2} H \Phi d \mu\right]\left[\int_{M} H \Phi d \mu\right] } \\
\leq & n\left[\int_{M} \tau_{i}^{2}\left|\mathcal{S}+\operatorname{Hess} h-\frac{g}{2 \tau_{i}}\right|^{2} H \Phi d \mu\right]\left[\int_{m} H \Phi d \mu\right] .
\end{aligned}
$$

Since

$$
\lim _{\tau_{i} \rightarrow 0} \int_{M} H \Phi d \mu=\Phi(y, T)<\infty
$$

and $\frac{1}{2} \mathcal{D}_{\alpha}(\nabla h) \geq 0$, we derive that

$$
\lim _{\tau_{i} \rightarrow 0} \int_{M} \tau_{i}\left(S+\triangle h-\frac{n}{2 \tau_{i}}\right) H \Phi d \mu=0 .
$$

Therefore, by Lemma 3.4,

$$
\begin{aligned}
\lim _{t \rightarrow T} \rho_{\Phi}(t) & =\lim _{\tau_{i} \rightarrow 0} \int_{M}\left[\tau_{i}\left(2 \triangle h-|\nabla h|^{2}+S\right)+h-n\right] H \Phi d \mu \\
& =\lim _{\tau_{i} \rightarrow 0} \int_{M}\left[\tau_{i}\left(\triangle h-|\nabla h|^{2}\right)+h-\frac{n}{2}\right] H \Phi d \mu \\
& =\lim _{\tau_{i} \rightarrow 0}\left[\int_{M}-\tau_{i} H \triangle \Phi d \mu+\int_{M}\left(h-\frac{n}{2}\right) H \Phi d \mu\right] \\
& =\lim _{\tau_{i} \rightarrow 0} \int_{M}\left(h-\frac{n}{2}\right) H \Phi d \mu \leq 0 .
\end{aligned}
$$

So $\beta \leq 0$. To show that equality holds, we proceed by contradiction. Without loss of generality, we may assume $\Phi(y, T)=1$. Let $H \Phi=$ $(4 \pi \tau)^{-n / 2} e^{-\tilde{h}}$ (that is, $\left.\tilde{h}=h-\ln \Phi\right)$, then integrating by parts yields,

$$
\rho_{\Phi}(t)=\mathbb{W}_{\alpha}(g, \tau, \tilde{h})+\int_{M}\left(\tau\left(\frac{|\nabla \Phi|^{2}}{\Phi}\right)-\Phi \ln \Phi\right) H d \mu
$$


By the choice of $\Phi$ the last term converges to 0 as $\tau \rightarrow 0$. So if $\lim _{t \rightarrow T} \rho_{\Phi}(t)=\beta<0$ then $\lim _{\tau \rightarrow 0} \mu_{\alpha}(g, \tau)<0$ and, thus, contradicts Lemma 2.7. Therefore $\beta=0$.

Now Theorem 1.2 follows immediately.

Proof. (Theorem 1.2) Recall from inequality (3.22)

$$
\partial_{t} \int_{M} v \Phi d \mu=\int_{M}\left(\square \Phi v-\Phi \square^{*} v\right) d \mu \geq 0 .
$$

By Proposition 3.5,

$$
\lim _{t \rightarrow T} \int_{M} v \Phi d \mu=0
$$

Since $\Phi$ is arbitrary, $v \leq 0$.

\section{REFERENCES}

[1] Bennett Chow, Sun-Chin Chu, David Glickenstein, Christine Guenther, James Isenberg, Tom Ivey, Dan Knopf, Peng Lu, Feng Luo, and Lei Ni. The Ricci flow: techniques and applications. Part III. Mathematical Surveys and Monographs. American Mathematical Society, Providence, RI. Geometric-Analysis aspects.

[2] Bennett Chow, Sun-Chin Chu, David Glickenstein, Christine Guenther, James Isenberg, Tom Ivey, Dan Knopf, Peng Lu, Feng Luo, and Lei Ni. The Ricci flow: techniques and applications. Part II, volume 144 of Mathematical Surveys and Monographs. American Mathematical Society, Providence, RI, 2008. Analytic aspects.

[3] Bennett Chow, Peng Lu, and Lei Ni. Hamilton's Ricci flow, volume 77 of Graduate Studies in Mathematics. American Mathematical Society, Providence, RI, 2006.

[4] Leonard Gross. Logarithmic sobolev inequalities and contractivity properties of semigroups. In Dirichlet forms, pages 54-88. Springer, 1993.

[5] Hongxin Guo and Tongtong He. Harnack estimates for geometric flows, applications to Ricci flow coupled with harmonic map flow. Geom. Dedicata, 169:411-418, 2014.

[6] Hongxin Guo, Robert Philipowski, and Anton Thalmaier. Entropy and lowest eigenvalue on evolving manifolds. Pacific J. Math., 264(1):61-81, 2013.

[7] Reto Müller. Monotone volume formulas for geometric flows. J. Reine Angew. Math., 643:39-57, 2010.

[8] Lei Ni. The entropy formula for linear heat equation. J. Geom. Anal., 14(1):87100, 2004.

[9] Lei Ni. A note on Perelman's LYH-type inequality. Comm. Anal. Geom., 14(5):883-905, 2006.

[10] Grisha Perelman. The entropy formula for the Ricci flow and its geometric applications. preprint, 2002.

[11] Natasha Sesum, Gang Tian, and Xiao-Dong Wang. Notes on perelmans paper on the entropy formula for the ricci flow and its geometric applications. preprint, 2003. 
[12] Hung Tran. Harnack estimates for ricci flow on a warped product. preprint arXiv:1211.6448, 2012.

[13] Qi S. Zhang. Bounds on volume growth of geodesic balls under Ricci flow. Math. Res. Lett., 19(1):245-253, 2012.

Department of Mathematics, Cornell University, Ithaca, NY 14853

E-mail address: cao@math.cornell.edu

SCHOOL OF MAThematics AND INFORMation SCIENCE, WenZhou UniverSity, Wenzhou, ZhejIANG 325035, China.

E-mail address: guo@wzu.edu.cn

Department of Mathematics, University of California, Irvine, CA 92697

E-mail address: hungtt1@math.uci.edu 